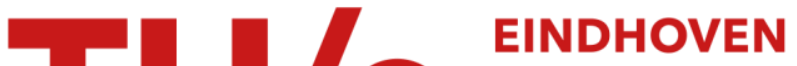 UNIVERSITY OF TECHNOLOGY
}

\section{Reconfiguring nanocomposite liquid crystal polymer films with visible light}

\section{Citation for published version (APA):}

Hauser, A. W., Liu, D., Bryson, K. C., Hayward, R. C., \& Broer, D. J. (2016). Reconfiguring nanocomposite liquid crystal polymer films with visible light. Macromolecules, 49(5), 1575-1581.

https://doi.org/10.1021/acs.macromol.6b00165

\section{Document license:}

TAVERNE

DOI:

10.1021/acs.macromol.6b00165

Document status and date:

Published: 09/03/2016

\section{Document Version:}

Publisher's PDF, also known as Version of Record (includes final page, issue and volume numbers)

\section{Please check the document version of this publication:}

- A submitted manuscript is the version of the article upon submission and before peer-review. There can be important differences between the submitted version and the official published version of record. People interested in the research are advised to contact the author for the final version of the publication, or visit the $\mathrm{DOI}$ to the publisher's website.

- The final author version and the galley proof are versions of the publication after peer review.

- The final published version features the final layout of the paper including the volume, issue and page numbers.

Link to publication

\section{General rights}

Copyright and moral rights for the publications made accessible in the public portal are retained by the authors and/or other copyright owners and it is a condition of accessing publications that users recognise and abide by the legal requirements associated with these rights.

- Users may download and print one copy of any publication from the public portal for the purpose of private study or research.

- You may not further distribute the material or use it for any profit-making activity or commercial gain

- You may freely distribute the URL identifying the publication in the public portal.

If the publication is distributed under the terms of Article $25 \mathrm{fa}$ of the Dutch Copyright Act, indicated by the "Taverne" license above, please follow below link for the End User Agreement:

www.tue.nl/taverne

Take down policy

If you believe that this document breaches copyright please contact us at:

openaccess@tue.nl

providing details and we will investigate your claim. 


\title{
Reconfiguring Nanocomposite Liquid Crystal Polymer Films with Visible Light
}

\author{
Adam W. Hauser, ${ }^{\dagger}$ Danqing Liu, ${ }^{\dagger}$ Kyle C. Bryson, ${ }^{\dagger}$ Ryan C. Hayward, ${ }^{*}{ }^{\dagger}$ and Dirk J. Broer ${ }^{*}, \S$ \\ ${ }^{\dagger}$ Department of Polymer Science \& Engineering, University of Massachusetts Amherst, Amherst, Massachusetts 01003, United States \\ ${ }^{\ddagger}$ Department of Chemical Engineering \& Chemistry and ${ }^{\S}$ Institute for Complex Molecular Systems, Eindhoven University of \\ Technology, 5600 MB Eindhoven, The Netherlands
}

\section{Supporting Information}

ABSTRACT: Patterns of white light are projected on liquid crystal (LC) polymer films containing gold nanospheres (NS) or nanorods (NR) to induce out-of-plane buckling through a photothermal effect. Straightforward synthetic techniques are used to provide well-dispersed nanocomposite films, with NRs exhibiting self-alignment with the LC director. Using a combination of prepatterned director orientation and spatiotemporal variations in light intensity, these nanocomposite films can be reversibly configured into different $3 \mathrm{D}$ states. Fine control over shape is demonstrated through variations in size, shape, and intensity of the illuminated region. Switching time scales are found to be of order a few seconds or below, likely reflecting the intrinsic relaxation time of the LC materials.
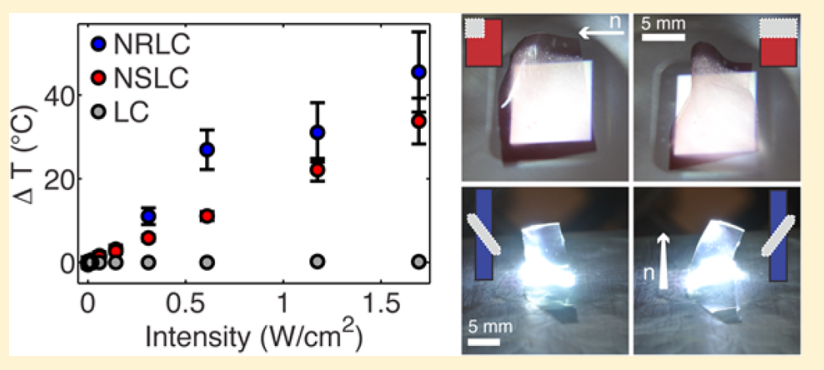

$\mathrm{P}$ rogrammed out-of-plane buckling of thin polymer sheets driven by in-plane patterns of distortion represents an exciting path toward adaptable materials systems. ${ }^{1-4}$ As such, several groups have demonstrated complex shape transformations from two-dimensional (2D) sheets into programmed three-dimensional (3D) forms relying on patterned in-plane expansion or contraction of hydrogels, ${ }^{5,6}$ shape memory materials, ${ }^{7}$ and liquid crystal (LC) networks. ${ }^{8-10}$ In most cases, however, only a single preprogrammed pathway can be followed from the initial flat configuration to the final buckled state. Multiple different 3D shape transitions within a single material have been demonstrated through write-eraserewrite processes based on exchange of ions, ${ }^{7,11}$ but this strategy has so far required the material be physically contacted and immersed in several different chemical environments to alter the programmed shape. Including independently addressable stimuli-responsive regions in a hydrogel, ${ }^{12,13}$ or embedding resistive microheaters in a thermally responsive network, ${ }^{14}$ can enable transitions between several shapes, but the number of different materials or independently controlled heaters necessary to access a wide range of $3 \mathrm{D}$ shapes becomes prohibitively complex. Polydomain LC networks containing a majority of azobenzene can bend along arbitrary directions specified by the polarization of illuminating ultraviolet (UV) light, but this strategy does not provide access to deformation modes more complex than a simple bend. ${ }^{15}$

Recently, photochemical ${ }^{16}$ or photothermal ${ }^{17,18}$ patterning of swelling in hydrogels has been pursued as a route to materials with arbitrarily reprogrammable 3D shapes. This approach, however, requires exchange of water with the surrounding environment to allow shape changes, and the elastic moduli of such hydrogels are typically quite low $(\sim 10-100 \mathrm{kPa})$, limiting the actuation forces that may be achieved. By contrast, monodomain LC networks undergo thermally induced decreases in nematic order parameter, enabling changes in shape without swelling by small molecules, and moduli ranging from $\mathrm{MPa}$ to $\mathrm{GPa}$ are possible by controlling the cross-link density and glass transition temperature. ${ }^{19,20}$ Inspired by these advantageous properties, a large body of work has focused on LC networks containing photoisomerizable azobenzene moieties as a means to photochemically reduce ordering and drive shape changes. ${ }^{21,22}$ While an attractive means for actuation, UV light is generally necessary to drive trans-cis isomerization, and the reverse reaction is slow unless a second stimulus (heat or a second light source) is used. Further, this mechanism has not yet been used to drive multiple 3D shape transitions from a freestanding single sheet of material. In a similar fashion, rubbery LC networks containing carbon nanotubes have allowed for photothermal actuation with visible or near-infrared light, but the changes in shape achieved have so far been limited to homogeneous photoinduced contraction of samples with mechanically induced planar alignment. ${ }^{23-26}$ Additionally, microparticles of magnetically aligned elastomeric LC polymers loaded with gold nanoparticles have been shown to enable localized photothermal bending through a two-photon absorption laser scanning method. ${ }^{27,28}$ However, these studies have so far been limited to rodlike particles with axial alignment of the director submersed within a liquid medium, and under

Received: January 24, 2016

Revised: February 10, 2016

Published: February 26, 2016 

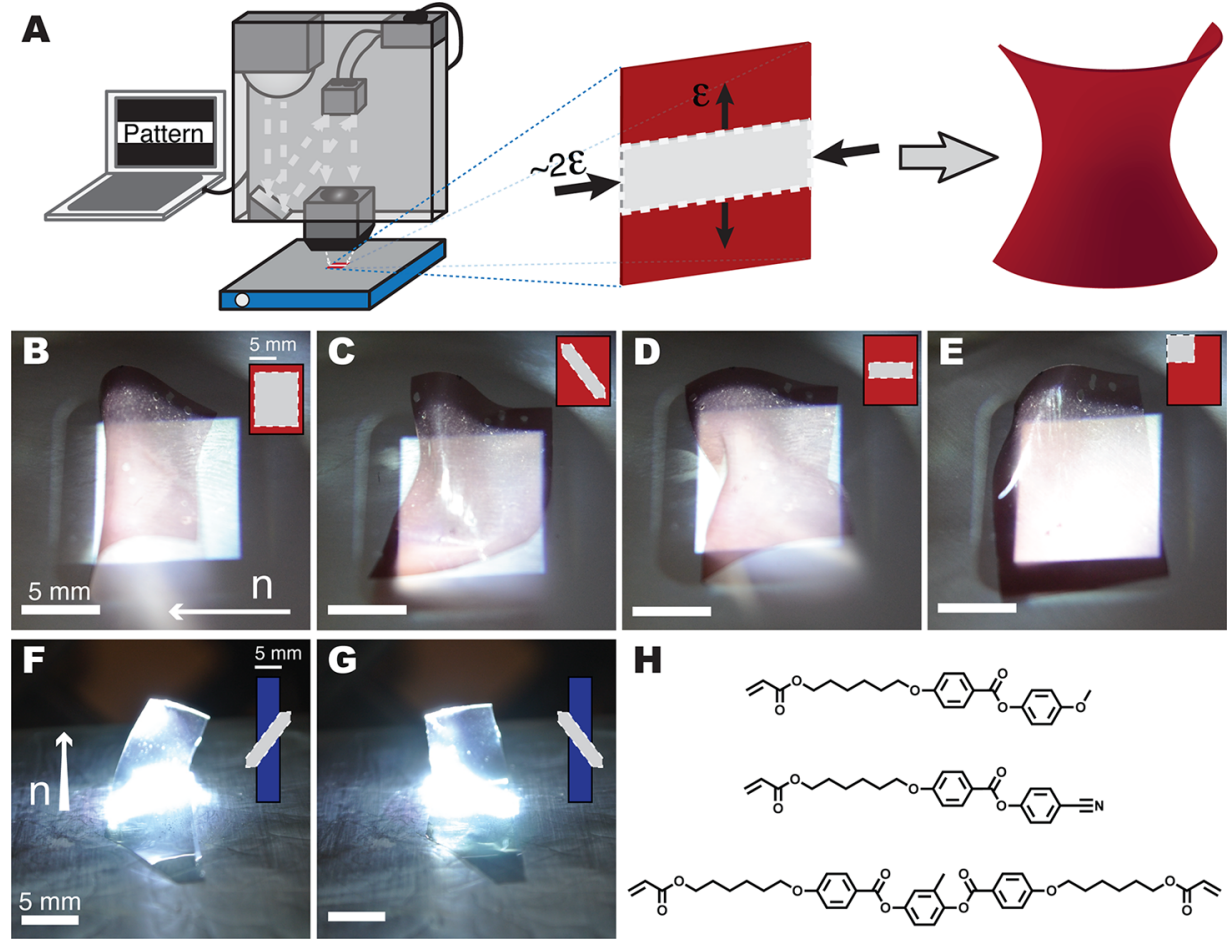

Figure 1. (A) Schematic representation of projector setup and in-plane strains induced on a planar nematic aligned thin film. (B-E) Selected shape transformations of planar oriented NSLC films; photos were taken less than $0.5 \mathrm{~s}$ after the projected patterns were turned off, and the background rectangle still present represents the projector's interpretation of $0 \%$ gray. (F, G) NRLC splay oriented films during illumination; a top view of the projected patterns on the samples is shown schematically and scaled down $3 \times$ in the top right corner of each photo. (H) Chemical structures of (top to bottom) RM105, RM23, and RM82.

certain scanning conditions the particles show nonreversible deformation.

Here, we introduce a simple approach to highly adaptable nanocomposite liquid crystal (NCLC) films that can be reversibly reconfigured through photothermal patterning of the reduction in order parameter. Using a modified commercial projector, we shine patterns of white light on monodomain liquid crystal polymer films containing gold nanospheres or nanorods (NSLC or NRLC, respectively) to locally induce inplane strains that can be controlled spatially and temporally (Figure 1A) with no additional stimuli needed for rapid and reversible actuation. In addition, the NRLC samples show selfalignment of NRs with the molecular liquid crystal director without any additional driving forces for alignment (i.e., stretching, or electric/magnetic fields), thus paving the way for controlled complex nanorod alignment in solid-state materials.

\section{RESULTS AND DISCUSSION}

The LCNC films are fabricated using routine LC alignment and photopolymerization methods. Briefly, we dissolve the monomer mixture (Figure $1 \mathrm{H})$ with the gold NS or NR $(0.4$ or $0.2 \mathrm{wt} \%$ with respect to monomers) and initiator in a cosolvent, evaporate the solvent above the nematic to isotropic transition temperature $\left(T_{\mathrm{N} / \mathrm{I}}\right)$, cast the isotropic mixture between two glass slides coated with rubbed polyimide, cool to $\sim 10{ }^{\circ} \mathrm{C}$ below $T_{\mathrm{N} / \mathrm{l}}$, and expose to $365 \mathrm{~nm}$ radiation for 10 min. Thiol end-capped poly(ethylene glycol) (PEG) with a number-average molecular weight of $800 \mathrm{~g} / \mathrm{mol}$ is used as a ligand for both NS and NR, providing good dispersion within the isotropic and nematic states of the NCLC mixtures before and after polymerization. The photoactuation experiments are performed with a digital micromirror based commercial projector with the color wheel removed and its lens reversed to focus the light down to the centimeter scale. Samples are placed on a hot plate with a temperature of $35{ }^{\circ} \mathrm{C}$ unless otherwise noted.

In Figure $1 \mathrm{~B}-\mathrm{E}$ we summarize the reversible buckling behavior of a NSLC film with planar director orientation, $\mathbf{n}$. In illuminated regions, the photothermally induced increase in temperature causes a reduction in the nematic order parameter, leading to in-plane shrinkage by roughly $2 \varepsilon$ along $\mathbf{n}$ and expansion by $\varepsilon$ in the perpendicular directions. To accommodate these localized strains, the films buckle out of plane, as it is energetically favorable to bend rather than stretch in thin films. Illuminating central horizontal strips (B, D), a diagonal strip (C), or a single corner (E) demonstrates that the reduction in order can be localized through photothermal patterning to create a variety of buckled configurations through minimization of the bending and stretching energies. The horizontal strips create axisymmetric "hourglass" rolled shapes, and the bottleneck region decreases with strip width $(\sim 10 \mathrm{~mm}$ in $\mathrm{B}$, compared to $\sim 3 \mathrm{~mm}$ in $\mathrm{D}$ ). The diagonal projection develops into a helix-like form with the corners bending in opposing directions, and a single corner illuminated drives that corner to fold. This represents the first demonstration of a single thin sheet of LC material that can be optically reprogrammed into multiple different $3 \mathrm{D}$ shapes, beyond simply altering the axis of bending. It is worth noting that such forms would not occur if there were a large gradient in absorbance through the thickness of the films, creating a gradient in the order parameter reduction. Highly doped planar azobenzene based systems often rely on such gradients for deformation, but this is also what limits such systems to simply 

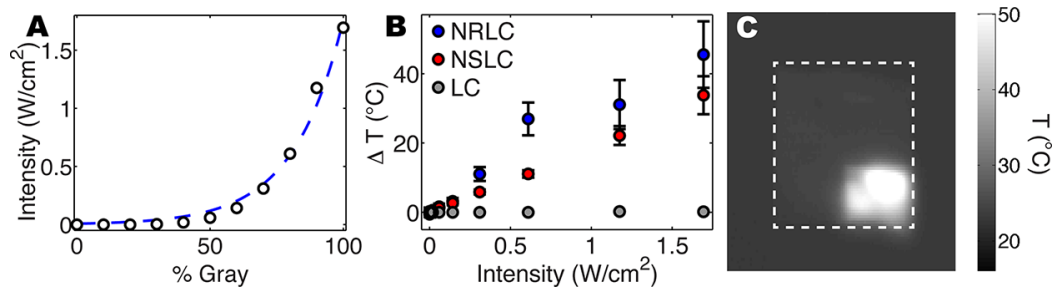

Figure 2. (A) Intensity versus projected gray values measured at $534 \mathrm{~nm}$ (circles) and single-exponential fit (dotted line); (B) the change in temperature observed via infrared camera of isotropic NR and NS and control (no particles) LC films versus intensity of projected light; (C) example infrared image of a small square pattern projected onto an isotropic film taken $\sim 30 \mathrm{~s}$ after illumination; the dotted line is a guide to the full area of the film and is $10 \mathrm{~mm}$ in width.
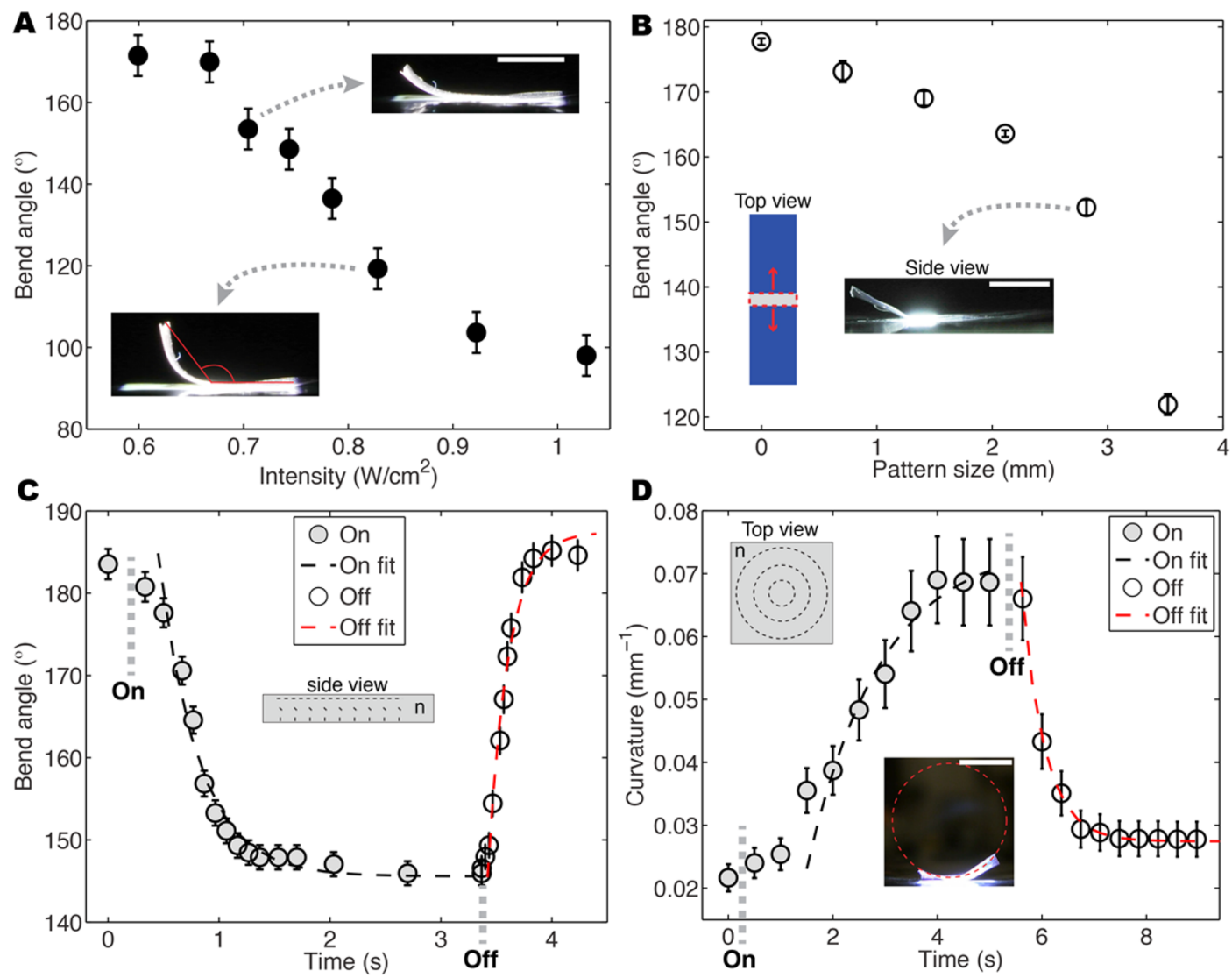

Figure 3. (A) Bend angle of a NSLC splay oriented film with intensity of projected light; light was projected over half of the sample length. (B) Bend angle with pattern width at a fixed intensity of $\sim 0.9 \mathrm{~W} \mathrm{~cm}^{-2}$ projected onto a NRLC splay film. (C, D) Bend or curvature with time of splay (C) and azimuthally (D) oriented NSLC films with single-exponential fits; insets are idealized schematics of the molecular order. Inset scale bars are all 10 $\mathrm{mm}$.

bend toward the light source. The high transmittance $(>80 \%$ from 450 to $600 \mathrm{~nm}$, Figure S4) in our planar films allows for in-plane strains to develop evenly through the thickness; thus, any given film was observed to buckle toward or away from the light, likely depending on the residual stress in the "light-off" state.

Figure 1F,G illustrates the power of combining more complex alignment in the LC film, splay in this case, ${ }^{29,30}$ with patterns of light to control the out-of-plane bending behavior. Upon a reduction in order parameter, the splay orientation exhibits a gradient in contraction through its thickness that mechanically acts as a "bilayer cantilever", thus yielding bending with mean curvature. ${ }^{1,31,32}$ Here, we show that patterned illumination allows both the location and orientation of this bending to be controlled.
The projector setup also enables facile control of the spatial intensity profile, simply through the grayscale value specified at each pixel. We find the measured intensity to have an exponential relationship with gray value (Figure 2A). To understand how intensity correlates with film temperature, we prepare control NCLC films by polymerizing in the isotropic state, such that photothermal heating does not drive significant buckling. Figure 2B shows the average temperature of an illuminated region of isotropic NS, NR, and control LC films with the measured intensity values, illustrating an expected linear trend of temperature change $(\Delta T)$ with intensity in the NCLCs, while the LC film lacking particles shows a negligible temperature change. Infrared measurements reveal that the local temperature increase can be tuned up to about $40{ }^{\circ} \mathrm{C}$, which reduces the order parameter of the LC film sufficiently to drive appreciable deformation. ${ }^{19}$ This localized order parameter 

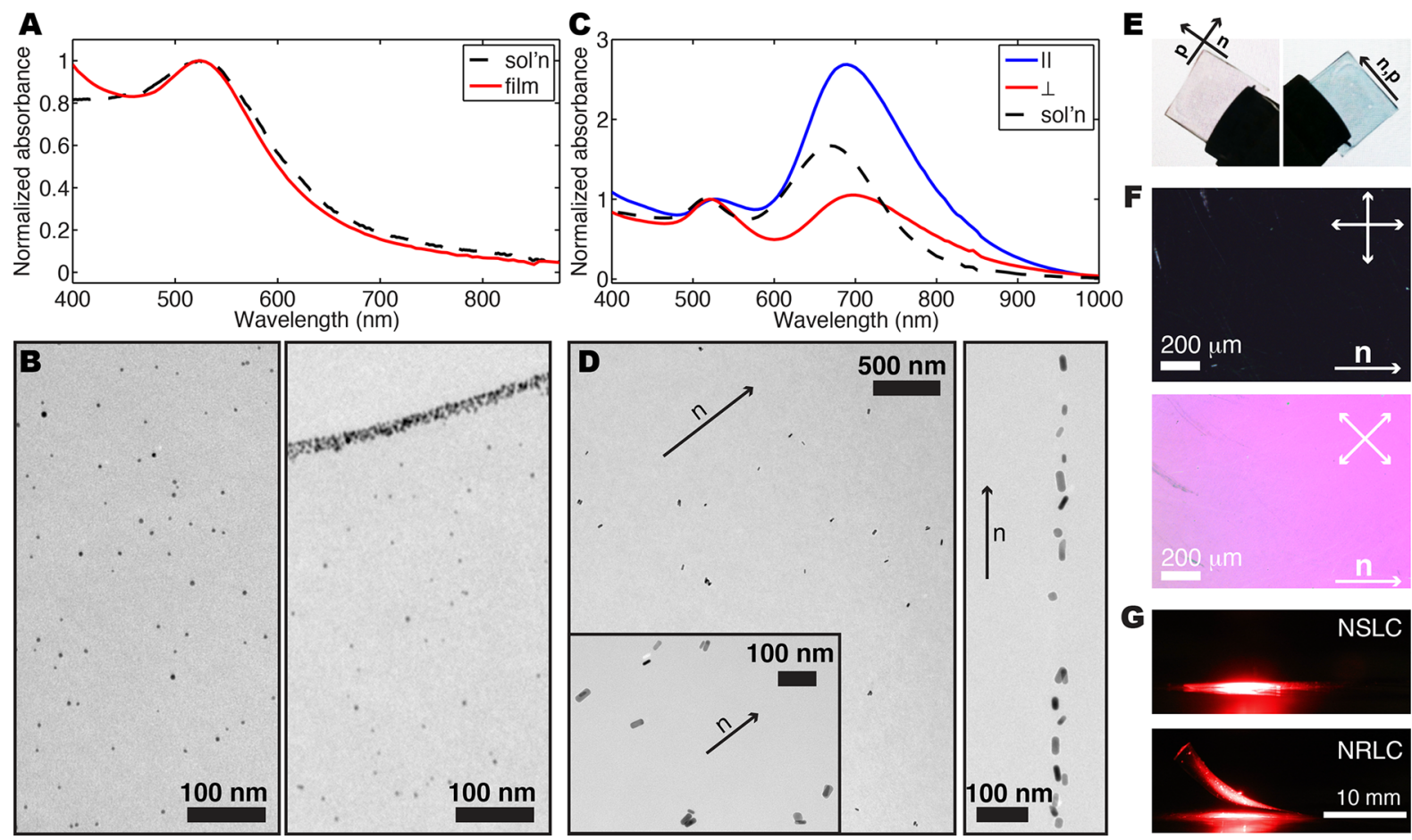

Figure 4. (A) Representative NSLC film and solution UV-vis spectra. (B) Cross-sectional TEM images of an NSLC film in the bulk (left) and at a surface (right). (C) Representative UV-vis spectra of a planar aligned NRLC film with polarization of incident light parallel and perpendicular to the director and a solution spectrum. (D) Cross-sectional TEM images of a planar aligned NRLC film in the bulk (left) and at a surface (right). (E) Digital photos of $1 \times 1 \mathrm{~cm}$ NRLC films in front of a single polarizer (black area is a tweezers). (F) POM images of nanocomposite films between cross polarizers. (G) Illumination of splay oriented NS (top) and NR (bottom) LCs with $650 \mathrm{~nm}$ light $\left(\sim 350 \mathrm{~mW} / \mathrm{cm}^{2}\right)$ at $50{ }^{\circ} \mathrm{C}$ starting temperature. The films are $\sim 30 \mu \mathrm{m}$ thick and contain 0.4 or $0.2 \mathrm{wt} \% \mathrm{NS}$ or NR, respectively. The microtomed slices are $\sim 60 \mathrm{~nm}$ thick.

reduction in conjunction with thermal softening allows for sufficiently large in-plane strains, and thus out-of-plane buckling, observed in our films. Further study is required to determine whether the switching kinetics are limited by heat transfer or the intrinsic network relaxation time.

We next consider the behavior of splay oriented LCNCs in more detail. Varying the magnitude of the contraction gradient or altering the in-plane size of the gradient region controls the mean curvature. Figure 3 shows that the bend angle can be finely tuned from flat to $90^{\circ}$ either by increasing light intensity with a constant size and shape of the illuminated region $(\mathrm{A})$ or by increasing the length of the illuminated region at constant intensity (B).

Analysis of the actuation in splay and azimuthally ${ }^{10}$ oriented films shows that the characteristic time scales, both forward and backward, are $\sim 1 \mathrm{~s}$ or less (Figure 3C,D). The characteristic actuation times are taken from single-exponential fits of bend angle or curvature with time and are found to be (light on/off) $0.5 / 0.2 \mathrm{~s}$ and $1.4 / 0.4 \mathrm{~s}$ for splay and azimuthally oriented samples, respectively. It is important to note that no second stimulus is needed to reverse the actuation, which is a significant advantage over systems based on UV-induced isomerization of azobenzene. Our actuation times are similar to those seen in azobenzene controlled systems, but the reversal of such systems generally requires actuation with a second light source of a different wavelength, or a much longer time in the dark. ${ }^{29,30,33}$ This allows for real-time control of shape with only a single light source, as exemplified in Videos SV1-4. Video SV1 shows light-controlled bending of a splay oriented film. Remarkably, as shown in Video SV2, the similar forward and backward switching speeds can lead to oscillatory behavior of splay-oriented films for appropriate intensities and sizes of the illuminated regions. We note that White and co-workers have studied a similar photoinduced oscillation of planar nematic films containing azobenzene, ${ }^{34,35}$ although the mechanism for oscillation differs somewhat. In their case, the film always bends toward the light, such that when the cantilever bends beyond $90^{\circ}$, the other side is exposed to light, driving bending in the opposite direction. Here, the splay oriented film always seeks to bend in the same direction; once the sample is actuated to $\sim 90^{\circ}$, it partially shadows the illuminated region and allows relaxation toward the flat state. Remarkably, both the amplitude and frequency of oscillation show variations in time, though a detailed understanding of this behavior will require further study. Videos SV3 and SV4 display two modes of actuation for azimuthally oriented films, controlled either by dynamically increasing the light intensity with a constant pattern (SV3) or by switching the location of a rectangular strip of constant intensity from one end to the other (SV4).

To achieve uniform and controlled photothermal actuation through NS or NR surface plasmon absorption, the nanoparticles must be reasonably well dispersed within the LC matrix. Several ligand chemistries are explored to meet this end, but $800 \mathrm{~g} / \mathrm{mol}$ PEG-thiol is found to provide the highest stability within the liquid and polymeric states. In contrast, aggregation is routinely observed in the polymerized films when particles are stabilized with higher molecular weight PEG-thiols ( 2 and $5 \mathrm{~kg} / \mathrm{mol}$ ) and small molecule ligands such as mercaptononanol, benzyl mercaptopropyl ether, ${ }^{36}$ hydroxyethyl methacrylate lipoic acid, ${ }^{37}$ and dodecanethiol. We suspect that the low molecular weight polymers are small enough to be partially wetted by the LC molecules but long enough to screen 
core-core attraction. PEG-thiol capped Au particles have been shown to provide stability within the nematic liquid state of 4cyano- $4^{\prime}$-pentylbiphenyl (5CB); ${ }^{38-40}$ to this point, we note that if polymerization is sufficiently fast compared to particle diffusion, the particles may be kinetically trapped in a dispersed state in the LC network.

The optical properties of gold nanoparticles are sensitive to interparticle distance, dielectric environment, shape, and size, ${ }^{41}$ providing a simple means to assess the dispersion state through ultraviolet-visible (UV-vis) absorption spectroscopy. Figure 4 shows the UV-vis spectra of planar NS and NR LC films and the solutions from which they were cast. The nearly identical spectra of the NSLC film and the solution reveal that the particles are generally well-dispersed throughout the bulk of the films-although some surface segregation is apparent via transmission electron microscopy (TEM, Figure 4B). UV-vis absorption of NRLC films with incident polarization parallel and perpendicular to the LC director clearly shows rod selfalignment but random spatial dispersion (Figure 4C); the alignment is apparent to the naked eye under a single polarizer as well (Figure 4F). Although alignment of NRs in small molecule LC media has been observed, ${ }^{38,39,42}$ to the best of our knowledge this is the first report of self-alignment of nanorods in LC polymer networks. Polarization-dependent UV-vis of both splay and azimuthal NRLC films show less pronounced, and no, polarization dependence, respectively, as shown in Figures S1 and S2. This is expected as the splay orientation exhibits a transition from planar to homeotropic alignment through its thickness and therefore only exhibits polarization dependence from the planar portion. A centered azimuthal sample captures the short axis of the NRs exclusively, but imperfect alignment, surface segregation, and/or imperfect sample symmetry with respect to incident light provide deviations from the ideal. The TEM images of NRLC films sliced through the thickness do show some surface segregation, and to fully understand the nature of the dispersion state of NS or NRs and to exploit the bulk self-alignment of the NRs requires a further separate study that is currently underway.

Finally, due to the size-dependent absorption of the nanoparticles, the NR and NSLCs are naturally wavelength selective. We demonstrate this simply in Figure 4G by shining a red LED on splay oriented NS and NRLCs to show that the NRLC actuates under $650 \mathrm{~nm}$ light, while the absorption in the NSLC case is insufficient to drive buckling even with twice the loading of $\mathrm{Au}$ nanoparticles, by weight.

\section{CONCLUSIONS}

We have demonstrated complex, reversible, and reprogrammable 3D buckling from monodomain nanocomposite liquid crystal polymer films through patterns of white light projected onto films with simple orientations of the nematic director. Finely controlled photothermal actuation is easily achieved through variations in both the shape of, and grayscale intensity patterns within, illuminated regions. The response times of both actuation and relaxation are on the order of a few seconds, and no additional stimulus is needed to to drive recovery. The ability to effectively reconfigure $3 \mathrm{D}$ shapes of these films is aided by the good dispersion of nanoparticles within the bulk of the LC networks. The gold nanorods show self-alignment with the liquid crystal director, which may provide the basis for interesting optical and optomechanical materials. The combination of structure on the nanoscale and dynamic patterning on the macro scale enables uniquely powerful routes to reconfigure
3D structure. Such systems can find applications in externally controlled soft robotics, plastic engines, and microfluidic systems.

\section{EXPERIMENTAL METHODS}

Materials. Poly(ethylene glycol) methyl ether thiol (PEG-SH, 800 $\mathrm{g} / \mathrm{mol}$ ), cetrimonium bromide ( $\mathrm{CTAB}, \geq 99 \%), \mathrm{HAuCl}_{4}$ trihydrate $(\geq 99.9 \%)$, ascorbic acid ( $\geq 99 \%)$, silver nitrate ( $\geq 99 \%), \mathrm{AuPPh}_{3} \mathrm{Cl}$, and borane tert-butylamine (97\%) were purchased from Sigma-Aldrich and used without further purification. Liquid crystal monomers RM105, RM82, and RM23 were purchased from Merck UK and used as received. Irgacure 819 photoinitiator was purchased from Ciba Specialty Chemicals, 2-( $N$-ethylperfluorooctanesulfonamido)ethyl methacrylate was purchased from Acros Organics, and polyimide $\mathrm{AL}$ 1051 was purchased from JSR Corp. and were used as received.

Nanosphere Synthesis. The nanospheres were synthesized by methods reported elsewhere with minor modifications. ${ }^{43}$ Briefly, 25 $\mathrm{mg}$ ( 1 equiv) of $\mathrm{AuPPh}_{3} \mathrm{Cl}$ was dissolved in $5 \mathrm{~mL}$ of chloroform, followed by $80 \mathrm{mg}$ ( 2 equiv) of PEG-SH. The mixture was introduced to a preheated water bath at $55{ }^{\circ} \mathrm{C}$ for $\sim 1 \mathrm{~min}$, then $43 \mathrm{mg}$ ( 10 equiv) of borane tert-butylamine was added as a powder, and the mixture was hand stirred until homogeneous. The reaction was allowed to sit unstirred at $55{ }^{\circ} \mathrm{C}$ for $2 \mathrm{~h}$; the result was a dark red solution. In a 50 $\mathrm{mL}$ centrifuge tube, $10 \mathrm{~mL}$ of ethanol followed by slow addition of $\sim 30 \mathrm{~mL}$ of hexanes (until solution turns purple and slightly turbid) was added and then centrifuged at $4000 \mathrm{rpm}$ for $30 \mathrm{~min}$, and the supernatant was poured off. About $1 \mathrm{~mL}$ of ethanol was added to the concentrate to redissolve, and then $2-5 \mathrm{~mL}$ of hexanes was added until turbid, followed by slow addition of chloroform until turbidity and red color returned, and finally slow addition of hexanes until slight turbidity and color change was seen again. The mix was centrifuged again and the supernatant poured off. The purified PEG nanoparticles were dissolved and stored in dichloromethane before use in LC systems.

Nanorod Synthesis. CTAB-stabilized gold nanorods were synthesized by a UV-assisted method reported elsewhere, ${ }^{44}$ followed by a ligand exchange procedure with PEG-SH. All reaction stocks were prepared with deionized water, and the CTAB stock was allowed to sit at $35^{\circ} \mathrm{C}$ for at least $30 \mathrm{~min}$ before use. Reaction solutions were made by adding stocks and solvents to a vial sequentially: $4.85 \mathrm{~mL}$ of CTAB $(80 \mathrm{mM}), 0.1 \mathrm{~mL}$ of acetone, $0.075 \mathrm{~mL}$ of cyclohexane, $0.05 \mathrm{~mL}$ of $\mathrm{AgNO}_{3}(10 \mathrm{mM}), 0.3 \mathrm{~mL}$ of ascorbic acid $(40 \mathrm{mM})$. Upon ascorbic acid addition, the solution turned colorless within $\sim 10 \mathrm{~s}$. The solution was distributed in $1 \mathrm{~mL}$ portions among $1 \mathrm{~cm}$ path length unfrosted quartz cuvettes and placed in a UV-B box for $1 \mathrm{~h}$. The resulting solutions were dark blue in color. The solutions were centrifuged at $13200 \mathrm{rpm}$ for $30 \mathrm{~min}$, and the clear top portion of water was decanted off $(2 \times)$. At least $2 \mathrm{~mL}$ of $4 \mathrm{mM}$ PEG-SH aqueous solution was added to the highly concentrated NRs, vortexed, and allowed to sit for $\sim 24 \mathrm{~h}$. The NRs were again centrifuged, and the clear portion was decanted off. Dichloromethane $(2 \mathrm{~mL})$ was added to the concentrated now PEG-SH coated NRs, vortexed, and allowed to completely transfer phases overnight. The solutions were again centrifuged, $2 \mathrm{~mL}$ of fresh dichloromethane was added to the concentrate, and then centrifuged one last time to obtain pure PEG-SH coated NRs.

LCNC Synthesis. A stock solution of the LC mixture containing 46 wt \% RM105, 29 wt \% RM82, 17 wt \% RM23, 1 wt \% 2-(Nethylperfluorooctanesulfonamido)ethyl methacrylate, and 1 wt \% Irgacure 819 was made in dichloromethane at $1 \mathrm{~g} / \mathrm{mL}$. NS or NR solutions were added at 0.4 or $0.2 \mathrm{wt} \%$ with respect to the LC solids, and the solvent was evaporated above the $T_{\mathrm{N} / \mathrm{I}}\left(\sim 72{ }^{\circ} \mathrm{C}\right)$ with intermittent vortexing. $20 \mu \mathrm{L}$ of the liquid was sandwiched between rubbed polyimide coated $3 \times 1 \mathrm{~cm}$ glass slides above $T_{\mathrm{N} / \mathrm{j}}$; the sample was transferred to a $50{ }^{\circ} \mathrm{C}$ surface for $1 \mathrm{~min}$ before exposing to $365 \mathrm{~nm}$ radiation $\left(\sim 30 \mathrm{~mW} / \mathrm{cm}^{2}\right)$ for $10 \mathrm{~min}$.

Projector Setup. An InFocus LP70+ projector with the color wheel removed and the projection optics reversed was used for all patterned illumination experiments. The light source was a $120 \mathrm{~W}$ high-pressure mercury lamp. 


\section{ASSOCIATED CONTENT}

\section{S Supporting Information}

The Supporting Information is available free of charge on the ACS Publications website at DOI: 10.1021/acs.macromol.6b00165.

UV-vis spectra of splay and azimuthally oriented NRLC films, dynamic mechanical analysis of a planar oriented NSLC film, the transmittance spectra of NS and NRLC films with unpolarized incident light (PDF)

Videos of actuations in real time (ZIP)

\section{AUTHOR INFORMATION}

\section{Corresponding Authors}

*E-mail hayward@umass.edu (R.C.H.).

*E-mail d.broer@tue.nl (D.J.B.).

\section{Funding}

The work in Eindhoven was funded by NWO Toppunt Project 718.014.003, while that at UMass was funded by the National Science Foundation through Grant EFRI-1332271.

\section{Notes}

The authors declare no competing financial interest.

\section{ACKNOWLEDGMENTS}

The authors thank Professor E. W. Meijer for helpful discussions and support, Tetsu Ouchi for help with DMA measurements, Professor Jae-Hwang Lee for the use of his infrared camera, and Dr. Louis Pitet for instigating this collaboration.

\section{REFERENCES}

(1) Chen, D.; Yoon, J.; Chandra, D.; Crosby, A. J.; Hayward, R. C. Stimuli-Responsive Buckling Mechanics of Polymer Films. J. Polym. Sci., Part B: Polym. Phys. 2014, 52, 1441-1461.

(2) Gracias, D. H. Stimuli Responsive Self-Folding Using Thin Polymer Films. Curr. Opin. Chem. Eng. 2013, 2, 112-119.

(3) Ionov, L. Polymeric Actuators. Langmuir 2015, 31, 5015-5024.

(4) Sharon, E.; Efrati, E. The Mechanics of Non-Euclidean Plates. Soft Matter 2010, 6, 5693-5704.

(5) Klein, Y.; Efrati, E.; Sharon, E. Shaping of Elastic Sheets by Prescription of Non-Euclidean Metrics. Science 2007, 315, 1116-1120.

(6) Kim, J.; Hanna, J. A.; Byun, M.; Santangelo, C. D.; Hayward, R C. Designing Responsive Buckled Surfaces by Halftone Gel Lithography. Science 2012, 335, 1201-1205.

(7) Kohlmeyer, R. R.; Buskohl, P. R.; Deneault, J. R.; Durstock, M. F.; Vaia, R. A.; Chen, J. Shape-Reprogrammable Polymers: Encoding, Erasing, and Re-Encoding. Adv. Mater. 2014, 26, 8114-8119.

(8) Ware, T. H.; McConney, M. E.; Wie, J. J.; Tondiglia, V. P.; White, T. J. Voxelated Liquid Crystal Elastomer Actuators. Science 2015, 347, 982-984.

(9) Lee, K. M.; Bunning, T. J.; White, T. J. Autonomous, Hands-Free Shape Memory in Glassy, Liquid Crystalline Polymer Networks. Adv. Mater. 2012, 24, 2839-2843.

(10) de Haan, L. T.; Sánchez-Somolinos, C.; Bastiaansen, C. M. W.; Schenning, A. P. H. J.; Broer, D. J. Engineering of Complex Order and the Macroscopic Deformation of Liquid Crystal Polymer Networks. Angew. Chem., Int. Ed. 2012, 51, 12469-12472.

(11) Palleau, E.; Morales, D.; Dickey, M. D.; Velev, O. D. Reversible Patterning and Actuation of Hydrogels by Electrically Assisted Ionoprinting. Nat. Commun. 2013, 4, 2257.

(12) Wu, Z. L.; Moshe, M.; Greener, J.; Therien-Aubin, H.; Nie, Z.; Sharon, E.; Kumacheva, E. Three-Dimensional Shape Transformations of Hydrogel Sheets Induced by Small-Scale Modulation of Internal Stresses. Nat. Commun. 2013, 4, 1586.
(13) Thérien-Aubin, H.; Wu, Z. L.; Nie, Z.; Kumacheva, E. Multiple Shape Transformations of Composite Hydrogel Sheets. J. Am. Chem. Soc. 2013, 135, 4834-4839.

(14) Yu, C.; Duan, Z.; Yuan, P.; Li, Y.; Su, Y.; Zhang, X.; Pan, Y.; Dai, L. L.; Nuzzo, R. G.; Huang, Y.; et al. Electronically Programmable, Reversible Shape Change in Two- and Three-Dimensional Hydrogel Structures. Adv. Mater. 2013, 25, 1541-1546.

(15) Ikeda, T. Photomodulation of Liquid Crystal Orientations for Photonic Applications. J. Mater. Chem. 2003, 13, 2037-2057.

(16) Kuksenok, O.; Balazs, A. C. Modeling the Photoinduced Reconfiguration and Directed Motion of Polymer Gels. Adv. Funct. Mater. 2013, 23, 4601-4610.

(17) Hauser, A. W.; Evans, A. A.; Na, J.-H.; Hayward, R. C. Photothermally Reprogrammable Buckling of Nanocomposite Gel Sheets. Angew. Chem., Int. Ed. 2015, 54, 5434-5437.

(18) Wang, E.; Desai, M. S.; Lee, S.-W. Light-Controlled GrapheneElastin Composite Hydrogel Actuators. Nano Lett. 2013, 13, 28262830.

(19) Liu, D.; Broer, D. J. Liquid Crystal Polymer Networks: Preparation, Properties, and Applications of Films with Patterned Molecular Alignment. Langmuir 2014, 30, 13499-13509.

(20) de Haan, L. T.; Schenning, A. P. H. J.; Broer, D. J. Programmed Morphing of Liquid Crystal Networks. Polymer 2014, 55, 5885-5896.

(21) Barrett, C. J.; Mamiya, J.; Yager, K. G.; Ikeda, T. PhotoMechanical Effects in Azobenzene-Containing Soft Materials. Soft Matter 2007, 3, 1249-1261.

(22) White, T. J.; Broer, D. J. Programmable and Adaptive Mechanics with Liquid Crystal Polymer Networks and Elastomers. Nat. Mater. 2015, 14, 1087-1098.

(23) Camargo, C. J.; Campanella, H.; Marshall, J. E.; Torras, N.; Zinoviev, K.; Terentjev, E. M.; Esteve, J. Localised Actuation in Composites Containing Carbon Nanotubes and Liquid Crystalline Elastomers. Macromol. Rapid Commun. 2011, 32, 1953-1959.

(24) Ji, Y.; Huang, Y. Y.; Rungsawang, R.; Terentjev, E. M. Dispersion and Alignment of Carbon Nanotubes in Liquid Crystalline Polymers and Elastomers. Adv. Mater. 2010, 22, 3436-3440.

(25) Li, C.; Liu, Y.; Lo, C.; Jiang, H. Reversible White-Light Actuation of Carbon Nanotube Incorporated Liquid Crystalline Elastomer Nanocomposites. Soft Matter 2011, 7, 7511.

(26) Yang, L.; Setyowati, K.; Li, A.; Gong, S.; Chen, J. Reversible Infrared Actuation of Carbon Nanotube-Liquid Crystalline Elastomer Nanocomposites. Adv. Mater. 2008, 20, 2271-2275.

(27) Sun, Y.; Evans, J. S.; Lee, T.; Senyuk, B.; Keller, P.; He, S.; Smalyukh, I. I. Optical Manipulation of Shape-Morphing Elastomeric Liquid Crystal Microparticles Doped with Gold Nanocrystals. Appl. Phys. Lett. 2012, 100, 241901.

(28) Evans, J. S.; Sun, Y.; Senyuk, B.; Keller, P.; Pergamenshchik, V. M.; Lee, T.; Smalyukh, I. I. Active Shape-Morphing Elastomeric Colloids in Short-Pitch Cholesteric Liquid Crystals. Phys. Rev. Lett. 2013, 110, 187802

(29) van Oosten, C. L.; Harris, K. D.; Bastiaansen, C. W. M.; Broer, D. J. Glassy Photomechanical Liquid-Crystal Network Actuators for Microscale Devices. Eur. Phys. J. E: Soft Matter Biol. Phys. 2007, 23, 329-336.

(30) van Oosten, C. L.; Bastiaansen, C. W. M.; Broer, D. J. Printed Artificial Cilia from Liquid-Crystal Network Actuators Modularly Driven by Light. Nat. Mater. 2009, 8, 677-682.

(31) Stoney, G. G. The Tension of Metallic Films Deposited by Electrolysis. Proc. R. Soc. London, Ser. A 1909, 82, 172-175.

(32) Timoshenko, S. Bending and Buckling of Bimaterial Strips. J. Opt. Soc. Am. 1925, 11, 233-255.

(33) Yamada, M.; Kondo, M.; Miyasato, R.; Naka, Y.; Mamiya, J.; Kinoshita, M.; Shishido, A.; Yu, Y.; Barrett, C. J.; Ikeda, T. Photomobile Polymer Materials-Various Three-Dimensional Movements. J. Mater. Chem. 2009, 19, 60-62.

(34) White, T. J.; Tabiryan, N. V.; Serak, S. V.; Hrozhyk, U. A.; Tondiglia, V. P.; Koerner, H.; Vaia, R. A.; Bunning, T. J. A High Frequency Photodriven Polymer Oscillator. Soft Matter 2008, 4, 1796-1798. 
(35) Serak, S.; Tabiryan, N.; Vergara, R.; White, T. J.; Vaia, R. A.; Bunning, T. J. Liquid Crystalline Polymer Cantilever Oscillators Fueled by Light. Soft Matter 2010, 6, 779-783.

(36) Hauser, A. W.; Hayward, R. C. Random Photografting of Polymers to Nanoparticles for Well-Dispersed Nanocomposites. J. Polym. Sci., Part B: Polym. Phys. 2016, 54, 152-158.

(37) Chen, X.; Lawrence, J.; Parelkar, S.; Emrick, T. Novel Zwitterionic Copolymers with Dihydrolipoic Acid: Synthesis and Preparation of Nonfouling Nanorods. Macromolecules 2013, 46, 119127.

(38) Senyuk, B.; Glugla, D.; Smalyukh, I. I. Rotational and Translational Diffusion of Anisotropic Gold Nanoparticles in Liquid Crystals Controlled by Varying Surface Anchoring. Phys. Rev. E - Stat. Nonlinear, Soft Matter Phys. 2013, 88, 1-11.

(39) Liu, Q.; Tang, J.; Zhang, Y.; Martinez, A.; Wang, S.; He, S.; White, T. J.; Smalyukh, I. I. Shape-Dependent Dispersion and Alignment of Nonaggregating Plasmonic Gold Nanoparticles in Lyotropic and Thermotropic Liquid Crystals. Phys. Rev. E - Stat. Nonlinear, Soft Matter Phys. 2014, 89, 1-10.

(40) Liu, Q.; Yuan, Y.; Smalyukh, I. I. Electrically and Optically Tunable Plasmonic Guest-Host Liquid Crystals with Long-Range Ordered Nanoparticles. Nano Lett. 2014, 14, 4071-4077.

(41) Eustis, S.; El-Sayed, M. A. Why Gold Nanoparticles Are More Precious than Pretty Gold: Noble Metal Surface Plasmon Resonance and Its Enhancement of the Radiative and Nonradiative Properties of Nanocrystals of Different Shapes. Chem. Soc. Rev. 2006, 35, 209-217.

(42) Zhang, Y.; Liu, Q.; Mundoor, H.; Yuan, Y.; Smalyukh, I. I. Metal Nanoparticle Dispersion, Alignment, and Assembly in Nematic Liquid Crystals for Applications in Switchable Plasmonic Color Filters. ACS Nano 2015, 9, 3097-3108.

(43) Zheng, N.; Fan, J.; Stucky, G. D. One-Step One-Phase Synthesis of Monodisperse Noble-Metallic Nanoparticles and Their Colloidal Crystals. J. Am. Chem. Soc. 2006, 128, 6550-6551.

(44) Niidome, Y.; Nishioka, K.; Kawasaki, H.; Yamada, S. Rapid Synthesis of Gold Nanorods by the Combination of Chemical Reduction and Photoirradiation Processes; Morphological Changes Depending on the Growing Processes. Chem. Commun. 2003, 23762377. 